\title{
Observation of Many New Argon Valence Satellites near Threshold
}

\author{
U. Becker, ${ }^{(a)}$ B. Langer, H. G. Kerkhoff, M. Kupsch, D. Szostak, and R. Wehlitz \\ Institut für Strahlungs - und Kernphysik, Technische Universität Berlin, D-1000 Berlin 12, West Germany \\ and \\ P. A. Heimann, ${ }^{(b)}$ S. H. Liu, D. W. Lindle, T. A. Ferrett, and D. A. Shirley \\ Materials and Chemical Sciences Division, Lawrence Berkeley Laboratory, Berkeley, California 94720 \\ (Received 8 September 1987)
}

\begin{abstract}
Photoelectron spectra and zero-volt electron scans were measured following synchrotron-radiation excitation of argon, to elucidate the photon energy range between threshold and the Cooper-minimum region of the Ar $3 s$ photoline. A number of the "correlation satellite" lines show a dramatic increase of fractional intensity, yielding a total of about forty observed photolines at threshold. This effect is interpreted as arising from strong interchannel coupling near threshold, yielding high-angular-momentum ionic states otherwise inaccessible. The distinction between "intrinsic" correlation effects due to discrete states and "dynamic" correlations arising through the photoemission process is emphasized.
\end{abstract}

PACS numbers: $33.60 . \mathrm{Cv}, 32.80 .-\mathrm{t}$

Satellite transition strengths are directly related to electron correlation effects associated with the photoionization process. A basic issue is the identification of the dominant correlation effect(s) contributing to the intensity of a given satellite. ${ }^{1-5}$ The energy region near threshold is particularly suited for differentiating among the various electron correlation mechanisms, which are expected to show different threshold behaviors. ${ }^{6-8}$

It is useful to distinguish between two types of correlations, which are, respectively, nearly independent of, and strongly dependent on, photon energy. ${ }^{9}$ The first type arises from "intrinsic" electron correlation effects in the neutral atom or the ionic core; the main corresponding correlations can be described by configuration interaction in the initial atom and final ion, and they would be present even in the absence of photoemission. Satellite intensities originating from these interactions reflect basically the spatial overlap between different configurations in the initial and ionic final states, ${ }^{10,11}$ modulated by transition-matrix elements. These intensities tend to vary slowly with photon energy.

In contrast, "dynamic " correlations exist explicitly through the dynamics of photoionization, and should therefore affect satellite intensities most strongly near threshold. The term "shake process" 12 is often used to describe that portion of the satellite intensity that arises indirectly, through the response of passive electrons, which fail to relax completely during photoionization. It is dynamic in the sense that it depends indirectly on the kinetic energy of the photoelectron tending to decrease near threshold, where the screening potential changes more slowly. Other aspects of dynamic correlations have been elaborated before in the context of continuum-state configuration interaction. ${ }^{13}$ The general feasibility of the separation of correlation effects by distinct threshold behavior has been demonstrated recently for different rare gases ${ }^{6,7}$ and molecules. ${ }^{8}$ We can think of continu- um-state configuration interaction as a virtual inelasticscattering process, in which the photoelectron interacts with the ion as it leaves, exchanging energy and angular momentum. This process should grow stronger near threshold. Inelastic scattering near threshold (continuum-state configuration interaction), while predicted for several cases,,${ }^{14}$ has been observed only in $\mathrm{He},{ }^{15}$ where it is the only possible type of correlation in the final state. The question arises: Does this continuum interaction, which is known to affect main-line intensities ${ }^{9}$ via the coupling of different subshells, also play a role for the satellite structure of atoms larger than helium?

Additional interest in the satellite near-threshold behavior has been stimulated by recent measurements of satellite cross-section effects observed earlier in main lines, such as autoionizing resonances, shape resonances, and Cooper minima. ${ }^{2,5,6,8}$ Such effects, if occurring in the threshold region, could be superimposed on the characteristic satellite intensities, leading to misinterpretations regarding the underlying correlation effects.

The valence subshells of Ar show such phenomena: a pronounced Cooper minimum, final-state interactions involving the continuum electron, ${ }^{9}$ and the beginning of an orbital collapse. ${ }^{16}$ The last leads to strong interactions in the final ionic state, which are assumed to govern the pronounced satellite structure of the $3 s$ photoline. ${ }^{17}$ The most prominent peak of this spectrum at higher photon energies, $3 s^{2} 3 p^{4} 3 d\left({ }^{2} S\right)$, was the subject of recent studies, ${ }^{3,4}$ over a wide energy range. However, none of these studies covered the energy range between the Cooper minimum and threshold.

In this Letter we report the first measurements of increasing fractional satellite intensity toward threshold in an atomic system with a nonhydrogenic final ionic state. We observed a dramatic increase in the number of $\mathrm{Ar}$ photoelectron satellite lines near threshold, in particular at zero kinetic energy. Our results for the 
$3 s^{2} 3 p^{4} 3 d\left({ }^{2} S\right)$ satellite show a pronounced Cooper minimum in the cross section of this satellite. The behavior of this satellite, despite a shifted Cooper minimum in the satellite channel, appears to be virtually constant down to threshold, as expected theoretically. ${ }^{11}$

We have studied the argon valence satellites in the photon energy range $32-100 \mathrm{eV}$, emphasizing the lower-energy end. The satellite peaks were assigned by comparison with corresponding optical levels. ${ }^{18}$ For our study we have selected some well-separated lines and groups of lines, labeled in order of their binding energies.

The experiments were performed at the Hamburger Synchrotronstrahlungslabor (HASYLAB) with use of a toroidal grating monochromator in conjunction with time-of-flight photoelectron analysis, and at the Stanford Synchrotron Radiation Laboratory (SSRL) with use of a spherical grating monochromator for a zero-volt electron scan. Both systems have already been described in some detail in earlier publications. ${ }^{6,7}$ The time-of-flight system was designed for high resolution and high transmission at low kinetic energies. With small acceleration voltages, the spectrometer, when optimally aligned, has a slowly changing transmission down to approximately 0.5 $\mathrm{eV}$; the overall resolution is about $2 \%$. The spectrometer used for the zero-volt electron scan is also based on time-of-flight detection, with an additional extraction field added across the interaction region. The resolution of the zero-volt spectrometer is better than $0.1 \mathrm{eV}$.

The main purpose of this investigation was to study the structure of the argon valence satellites at energies below the Cooper minimum of the $3 \mathrm{~s}$ main line, down to threshold. Figure 1 shows the absolute cross section of the well-known $3 s^{2} 3 p^{4} 3 d\left({ }^{2} S\right)$ "virtual-Auger"-type satellite versus photon energy, together with the data points of other authors, which were normalized to our $3 \mathrm{~s}$ cross-section curve. The dash-dotted curve in Fig. 1 represents this $3 s$ cross-section curve, scaled to our satellite intensity at high kinetic energies $(17.2 \%$ of $3 s$ at $80 \mathrm{eV})$. The satellite cross section shows the same qualitative behavior, except in the Cooper-minimum region, in good agreement with predictions of the many-body perturbation theory. ${ }^{11}$ A simple explanation of this deviation is that the Cooper minimum in the satellite channel is due to coupling with the $3 s$ main line, causing a Cooper minimum near but not exactly at the same photon energy. The dashed curve represents the same scaled $3 s$ cross section, shifted by roughly $3 \mathrm{eV}$ to higher photon energies. This curve fits the satellite cross section unexpectedly well, supporting the idea of a "shifted Cooper minimum" in the satellite channel.

The (satellite)/( $3 s$ main line) intensity ratios are strongly modulated near threshold by Cooper minima. However, comparison of the satellite and main-line intensities at equal kinetic energies shows an essentially constant ratio in many cases below the energy of the $3 \mathrm{~s}$ Cooper minimum. The Cooper minimum is in these

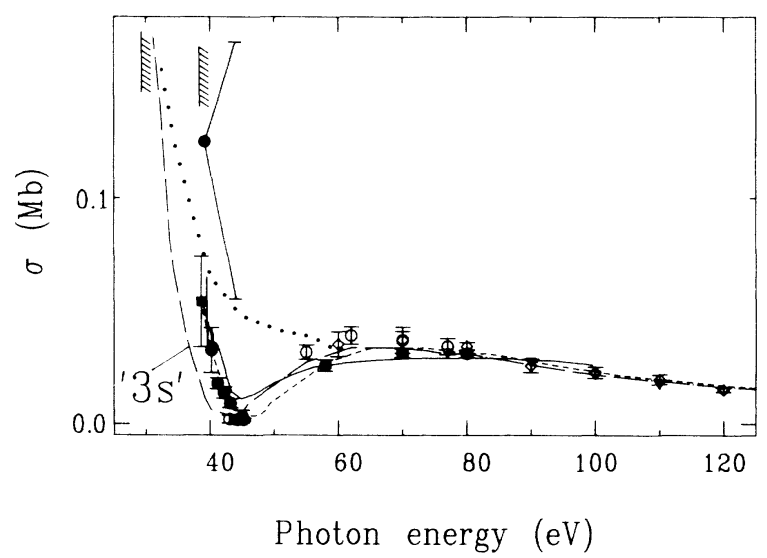

FIG. 1. Partial cross section of the Ar $3 s$ satellite $3 s^{2} 3 p^{4} 3 d\left({ }^{2} S\right)$ (satellite 4) as a function of the photon energy. The filled symbols (circles, time-of-flight photoelectron spectra; squares, zero-volt electron scan normalized by the assumption of $\sigma_{3 s}=1.5 \mathrm{Mb}$ as an average of different theoretical values) represent our results. The open circles are measurements of Adam, Morin, and Wendin (Ref. 2); the lozenges are from Brion, Bawagon, and Tan (Ref. 3); and the triangles are from Kossmann et al. (Ref. 4). The dash-dotted curved shows our $3 s$ cross-section data scaled to the satellite intensity at high kinetic energy; the dashed curve represents the same cross section but shifted by $3.35 \mathrm{eV}$ to higher photon energies. The dotted line is an arbitrarily selected monotonic curve which matches the $3 s$ cross section above and below the Cooper minimum. Its purpose is simply to illustrate the effect of the $3 \mathrm{~s}$ Cooper minimum on the satellite branching ratio, in Fig. 2. The solid line represents a many-body perturbation-theory calculation (Ref. 11).

cases only an additional effect, which obscures the general threshold behavior of a satellite. To visualize this effect, we have drawn a rough-and arbitrary-curve (dotted line) for a hypothetical $3 s$ cross section without a Cooper minimum. On dividing this curve by the actual $3 s$ cross section, we obtain a ratio which shows the general modulating effect of the $3 s$ Cooper minimum on a (satellite)/(main line) branching ratio.

In order to remove the kinetic-energy effect, Fig. 2 shows three selected (satellite)/( $3 s$ main line) ratios taken at equal kinetic energy, together with such a tentative ratio curve. This curve is scaled to the respective satellite intensities at high kinetic energy, both as a reminder of the Cooper-minimum effect and as a guide to delineate roughly the unaffected near-threshold region. In this near-threshold region we have observed basically two types of behavior. The first is a constant ratio, as shown by satellite 3: $\left({ }^{1} S\right) 4 s\left({ }^{2} S\right)$, which we have also seen in Fig. 1 for satellite $4,\left[\left({ }^{1} D\right) 3 d\left({ }^{2} S\right)\right]$. The second, largely unexpected, is an increasing fractional intensity towards threshold, as shown by satellite $2\left[\left({ }^{3} P\right) 3 d\left({ }^{4} D\right)\right.$, $\left.\left({ }^{3} P\right) 4 s\left({ }^{4} P\right)\right]$. Satellite 1 , consisting of two final ionic states $\left({ }^{1} D\right) 3 d\left({ }^{2} G\right)$ and $\left({ }^{3} P\right) 4 p\left({ }^{4} P^{\circ}\right)$, shows clear indications of resonance enhancement due to autoionization 


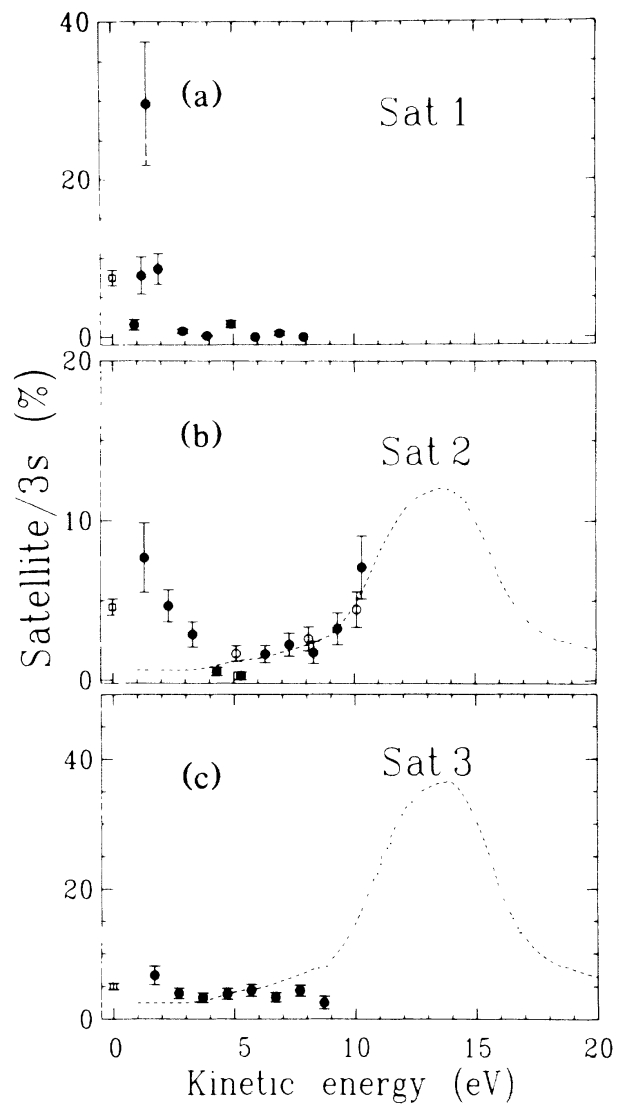

FIG. 2. Branching ratios of three selected satellites (satellites $1-3$ ) with respect to the $3 s$ main line at the same kinetic energy. The filled symbols are our results; the open circles are data from Adam, Morin, and Wendin (Ref. 2). The dashed line as explained in the text represents the effect of the $3 \mathrm{~s}$ Cooper minimum on the satellite to main-line ratio.

of discrete states (double excitations to $4 p 5 s$ states around ${ }^{19} 33.6 \mathrm{eV}$ ) into this satellite channel, an effect already observed for $\mathrm{He}$ (Ref. 15) and $\mathrm{Ne}^{6}{ }^{6}$ However, the enhancement structure for satellite 2 is different from discrete excitations. We propose that this threshold enhancement, which is also shown by other satellites in the Ar valence satellite spectrum, results from "virtual inelastic scattering" of the outgoing electron in the final state.

To support this interpretation of the observed threshold behavior, we show in Fig. 3 a sequence of three different photoelectron spectra taken in the nearthreshold region, together with a zero-volt electron scan. These spectra differ dramatically from all previous satellite spectra taken at higher photon energies, both in the number of resolved lines and in their intensity distribution. The zero-volt electron scan shows, in particular, that to almost every optically observed, excited $\mathrm{Ar}^{+}$level there corresponds a satellite line. This means that most of the excited $\mathrm{Ar}^{+}$configurations interact with the $3 \mathrm{~s}$

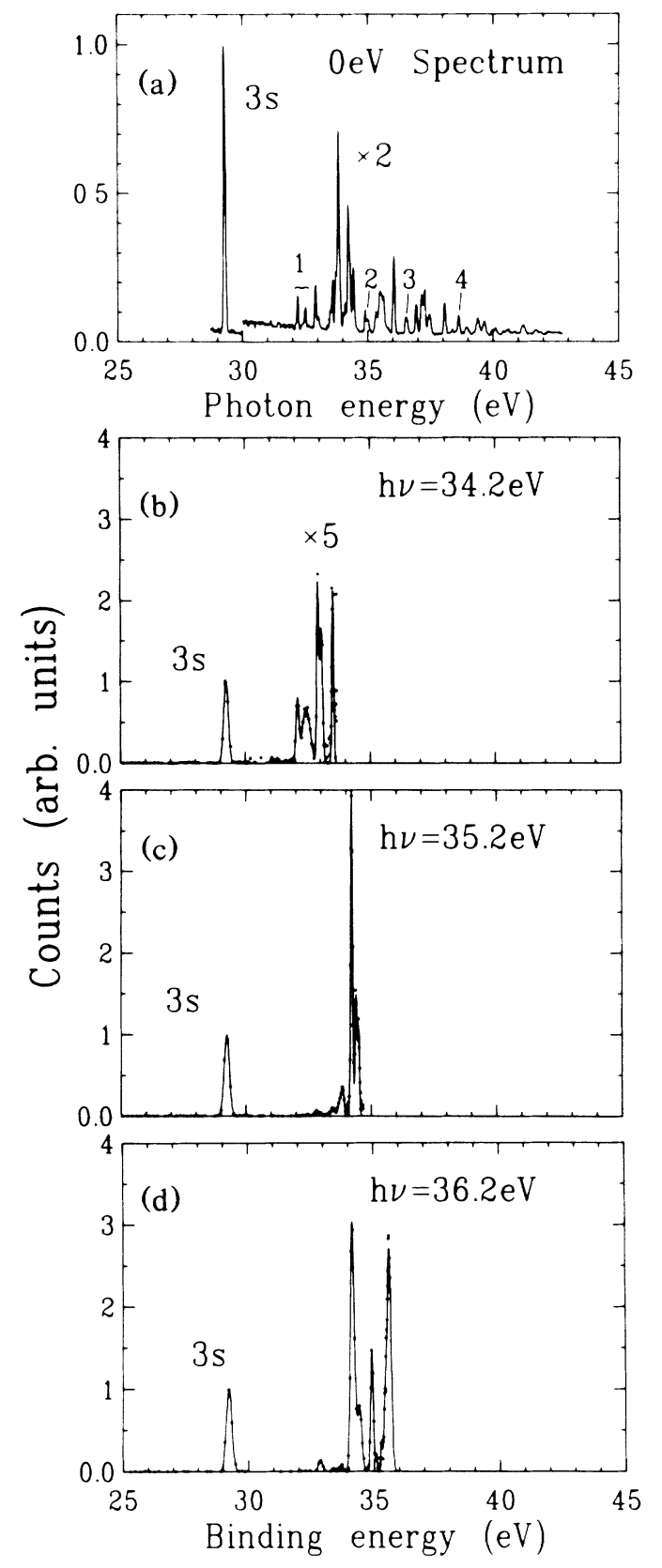

FIG. 3. (b)-(d) Photoelectron spectra taken in the nearthreshold region of the satellites with lower binding energy together with (a) a zero-volt electron scan between 29 and 43 $\mathrm{eV}$. The numbers in (a) show the position of those satellites which are shown in Figs. 1 and 2. Note that the satellite intensities in (c) and (d) are shown on normal scale.

and $3 p$ hole states which are genuinely associated with the Ar valence photoionization process. However, most of these satellite intensities decrease quickly with increasing photon energies.

How can we explain the qualitative difference between the satellite intensities at threshold and satellite spectra at higher photon energies? The straightforward designa- 
tion of most of the satellite peaks by optical data ${ }^{18}$ shows a large number of final ionic states such as ${ }^{2} G$ and ${ }^{4} F$, which cannot be populated directly through photoemission, by our invoking correlations only in the ground and final ionic states. To explain the intensities of these satellite states apart from accidental autoinization at threshold, final-state interactions must be taken into account. In the final state the photoelectron may be thought of as inelastically scattering on its way out, thereby exciting another electron. In the characterization of the energy dependence of these inelasticscattering processes, it is useful to consider the excess energy necessary for the excitation. If we compare $\mathrm{Ar}$ on an excess energy scale with $\mathrm{He}^{+}(2 p),{ }^{15}$ all satellites associated with a primary Ar $3 s$ hole should lose their intensity over a range of a few electronvolts. This behavior is shown, for example, by satellite 2 , having the main configuration $\left({ }^{1} D\right) 3 d\left({ }^{2} G\right)$, a result corroborated by very recent fluorescence measurements. ${ }^{20}$ This supports our interpretation of the threshold behavior of this satellite as arising through inelastic scattering.

The large overlap between the $3 s$ hole state and the states with a collapsed $3 d$ orbital, which all belong to the same manifold with $n=3,{ }^{21}$ is probably responsible for the strength of the observed inelastic-scattering processes. However, the strong coupling between the Ar $3 s$ and $3 p$ main lines, especially near threshold, ${ }^{9}$ partly obscures the usual distinction between a $3 s$ and a $3 p$ satellite in this region.

In conclusion, we have shown the importance of inelastic-scattering processes to the valence satellite structure of argon by studying the threshold and nearthreshold behavior of individual satellite lines. Our interpretation of the observed intensity enhancement near threshold is supported by the appearance of many previously unobserved satellite transitions which are not allowed by interactions in the initial and ionic final states alone. Clearly, more experimental and theoretical work is needed to understand these new phenomena.

We would like to thank Professor B. Sonntag and the members of his research group for their assistance and interest. This work was supported in part by the Bundesminister für Forschung und Technologie under Contract No. 05205 BK and in part by the Director, Office of Energy Research, Office of Basic Energy Sciences, Chemical Sciences Division of the U.S. Department of Energy under Contract No. DE-AC03-76SF00098. It was performed in part at the Stanford Synchrotron Radiation Laboratory, which is supported by the U.S.
Department of Energy, Office of Basic Energy Sciences.

(a) Present address: Physikalisches Institut, Universität Würzburg, Am Hubland, D-8700 Würzburg, West Germany.

(b) Present address: Physik-Department, Technische Universität München, D-8000 München, West Germany.

${ }^{\mathrm{l}} \mathrm{F}$. Wuilleumier, J. Phys. (Paris), Colloq. 43, C2-347 (1982), and reference therein.

${ }^{2}$ M. Y. Adam, P. Morin, and G. Wendin, Phys. Rev. A 31, 1426 (1985), and references therein.

${ }^{3}$ C. E. Brion, A. O. Bawagon, and K. H. Tan, Chem. Phys. Lett. 134, 76 (1987).

${ }^{4}$ H. Kossmann, B. Krässig, V. Schmidt, and J. E. Hansen, Phys. Rev. Lett. 58, 1620 (1987), and reference therein.

${ }^{5}$ A. Fahlman, M. O. Krause, T. A. Carlson, and A. Svensson, Phys. Rev. A 30,812 (1984).

${ }^{6}$ U. Becker, R. Hölzel, H. G. Kerkhoff, B. Langer, D. Szostak, and R. Wehlitz, Phys. Rev. Lett. 56, 1120 (1986), and references therein.

${ }^{7}$ P. A. Heimann, U. Becker, H. G. Kerkhoff, B. Langer, D. Szostak, R. Wehlitz, D. W. Lindle, T. A. Ferrett, and D. A. Shirley, Phys. Rev. A 34, 3782 (1986).

${ }^{8}$ A. Reimer, J. Schirmer, J. Feldhaus, A. M. Bradshaw, U. Becker, H. G. Kerkhoff, B. Langer, D. Szostak, R. Wehlitz, and W. Braun, Phys. Rev. Lett. 57, 1707 (1986).

${ }^{9}$ C. D. Lin, Phys. Rev. A 9, 171 (1974).

${ }^{10}$ H. Smid and J. E. Hansen, Phys. Rev. Lett. 52, 2138 (1984), and references therein.

${ }^{11}$ W. Wijesundera and H. P. Kelly, Phys. Rev. A 36, 4539 (1987).

${ }^{12}$ T. A. Carlson and M. O. Krause, Phys. Rev. 140, A1057 (1965); T. Aberg, Phys. Rev. 156, 35 (1967).

${ }^{13}$ S. T. Manson, J. Electron Spectrosc. Relat. Phenom. 9, 21 (1976), and references therein.

${ }^{14}$ K. A. Berrington, P. G. Burke, W. C. Fon, and K. T. Taylor, J. Phys. B 15, L603 (1982); T. Ishihara, J. Mizuno, and T. Watanabe, Phys. Rev. A 22, 1552 (1980).

${ }^{15}$ D. W. Lindle, T. A. Ferrett, U. Becker, P. H. Kobrin, C. M. Truesdale, H. G. Kerkhoff, and D. A. Shirley, Phys. Rev. A 31, 714 (1985), and reference therein.

${ }^{16}$ J. P. Connerade, Contemp. Phys. 19, 415 (1978); R. I. Karaziya, Usp. Fiz. Nauk. 135, 79 (1981) [Sov. Phys. Usp. 24, 775 (1981)].

${ }^{17}$ J. E. Hansen, Comments At. Mol. Phys. 12, 197 (1982).

${ }^{18}$ C. E. Moore, Atomic Energy Levels, NBS Special Publication No. 467 (U.S. GOP, Washington, DC, 1969), Vol. 1.

${ }^{19}$ R. P. Madden, D. L. Ederer, and K. Codling, Phys. Rev. 177, 136 (1969).

${ }^{20}$ J. A. R. Samson, Y. Chung, and E.-M. Lee, to be published.

${ }^{21}$ D. Layzer, Ann. Phys. (N.Y.) 8, 271 (1959); V. Schmidt, Z. Phys. D 2, 275 (1986). 